\title{
A Simple Model for the Anomalous Counterclockwise Turning of the Surface Wind with Time over the Great Plains of the United States
}

\author{
RICHARD ROTUNNO AND GLEN S. ROMINE \\ National Center for Atmospheric Research, Boulder, Colorado \\ HOWARD B. BLUESTEIN \\ School of Meteorology, University of Oklahoma, Norman, Oklahoma
}

(Manuscript received 1 March 2018, in final form 6 June 2018)

\begin{abstract}
A recent study found that surface hodographs over the Great Plains of the United States turn in a counterclockwise direction with time. This observed turning is opposite of the clockwise turning observed (and expected, based on theory) at higher altitudes. Using a mesoscale forecast model, the same study shows that it has the same hodograph behavior as found in the observations. The study further shows that the reason for this anomalous counterclockwise turning is the decoupling of the surface layer from the boundary layer after sunset and its recoupling after sunrise. The present paper presents a simple model for this behavior by extending a recent analytical model for the diurnal oscillation to include the surface-layer effect. In addition, selected solution features are analyzed in terms of several of the nondimensional input parameters.
\end{abstract}

\section{Introduction}

The nocturnal low-level jet over the Great Plains of the United States has been a noted feature of its warm-season climatology since the 1950s [see the comprehensive literature review in Shapiro et al. (2016), hereafter SFR]. Observations of the horizontal wind vector $(u, v)$ as a function of time at a fixed altitude plotted on a hodograph generally exhibit an orbit on which time increases in the clockwise direction (called "clockwise turning"). In a recent paper, Bluestein et al. (2018) found that such hodographs computed from observations at $10 \mathrm{~m}$ (considered the "surface") exhibit counterclockwise turning; moreover, mesoscalemodel forecasts averaged over the warm season of 2016 show the same feature. Analysis of the mesoscale-model hodographs some distance above the surface show the expected clockwise turning. The present study shows that an extension of the SFR model of diurnally varying boundary layer winds over sloping terrain to include a semislip (instead of a "no slip") lower-boundary condition can capture the anomalous counterclockwise turning of the surface wind for conditions typical of the Great Plains in the warm season.

\footnotetext{
Corresponding author: Richard Rotunno, rotunno@ucar.edu
}

In addition to the qualitative difference in the turning of the surface hodograph between models with semislip and no-slip lower-boundary conditions, there are quantitative differences in the wind profiles above the surface. To compare the present results with those of SFR, and to explore efficiently the parameter space, we will present most of the results in a nondimensional framework in which the SFR no-slip solutions emerge in the limit as the surface-drag coefficient becomes large.

In section 2, the SFR model is summarized and extended to have a semislip lower-boundary condition. As only numerical solutions are available to us, we first check the present numerical model results against the analytical solutions reported in SFR. A direct comparison of the latter against a semislip solution for the same set of external parameters shows counterclockwise turning of the surface wind is a result of the semislip condition. Quantitative differences noted in the hodographs above the surface motivate the dimensional analysis of section 3 , where several important nondimensional parameters are identified. Results for the maximum jet strength, its height, and its time of occurrence are tabulated as a function of the most important nondimensional input parameters of the present simple model. A summary is given in section 4 . 


\section{The model}

\section{a. Governing equations}

The governing equations are fully described in SFR, so only a brief summary is given here. In modified form, they are

$$
\begin{array}{ll}
\partial_{t} u=f\left(v-v_{G}\right)-b \sin \alpha & +\partial_{z} \tau_{x}, \\
\partial_{t} v=-f u & +\partial_{z} \tau_{y}, \\
\partial_{t} b=u N^{2} \sin \alpha-\delta b & +K \partial_{z z} b,
\end{array}
$$

where

$$
\left(\tau_{x}, \tau_{y}\right)=K \partial_{z}(u, v)
$$

is the shear stress, $b$ is the buoyancy, $N$ is the BruntVäisälä frequency, $f$ is the Coriolis parameter, $v_{G}$ is the geostrophic wind, $\delta$ is the radiative-damping coefficient, and $K(t)$ is the time-varying eddy viscosity and diffusivity. The terrain-slope angle $\alpha$ represents the rotation of the coordinate system such that the $x$ (east-west) axis aligns with the sloping plain, which is assumed to be invariant in the $y$ (north-south) direction (see Fig. 1 of SFR). The upper-boundary conditions are $\left(u, v-v_{G}, b\right) \rightarrow 0$ as the vertical coordinate $z \rightarrow \infty$. The lower-boundary conditions are

$$
\begin{aligned}
\left.b\right|_{z=0} & =\frac{\Delta b}{2} s(t), \\
\left.\left(\tau_{x}, \tau_{y}\right)\right|_{z=0} & =\left.C_{D} \sqrt{u^{2}+v^{2}}(u, v)\right|_{z=d},
\end{aligned}
$$

where $\Delta b$ represents the diurnal buoyancy (temperature) variation, $s(t)$ is a diurnally periodic function of unit amplitude, $C_{D}$ is the drag coefficient, and $d$ is within the surface layer. In SFR, the no-slip condition

$$
\left.(u, v)\right|_{z=0}=0
$$

is used instead of (6); we will show below that the solutions using (6) approach the no-slip solutions as $C_{D}$ becomes large.

The different lower-boundary conditions, (6) and (7), warrant a few words of explanation. In the case of (7) (no slip), the eddy viscosity defines the shear stress (4) at all heights, including $z=0$. In the case of (6) (semislip), the shear stress at $z=0$ is defined by the drag law (6) using the velocity at $z=d$, where $d$ is several meters above the actual lower boundary at $z=0$. The level $z=d$ is referred to as the "surface" in standard meteorological usage (Glickman 2000). In the following comparison of solutions using either (6) or (7), we follow standard practice and refer to the height $z=d$ as the surface with the understanding that in the semislip case, there is an implied logarithmic (constant stress) layer that brings the velocity to zero at $z=0$ (e.g., Lumley and Panofsky 1964, p. 103). In the following, we refer to (4) as the interior stress and (6) as the surface stress when discussing the semislip case.

Following SFR, we adopt a square wave for the time variation of $K(t)$, with a larger value $K_{d}$ during the day and a smaller value $K_{n}$ at night. The function $s(t)$ is given by a symmetric triangle wave of unit amplitude and frequency $\omega=2 \pi / 86400 \mathrm{~s}$. In the present study, sunrise is denoted by $t=0 \mathrm{~h}$; the phase of $s(t)$ with respect to $K(t)$ is adjusted such that the peak surface temperature occurs at $t=t_{\max }$ (and thus, the minimum occurs at $\left.t_{\max }-12 \mathrm{~h}\right)$, which is chosen to occur between noon $(t=6 \mathrm{~h})$ and sunset $(t=12 \mathrm{~h})$. In SFR, $s(t)$ is an asymmetric triangle wave chosen so that the surface temperature is minimum at $t=0 \mathrm{~h}$ (sunrise) and maximum at $t=t_{\max }$; we found the differences in solutions using symmetric and asymmetric $s(t)$ to be small, so to simplify the following dimensional analysis, we opted for the former. ${ }^{1}$

The SFR model contains the two most popular mechanisms proposed to explain the low-level jet in particular, and the diurnal wind oscillation in general, over the Great Plains. With $\alpha=0$ (no slope), the diurnal variation of the eddy viscosity $K(t)$ in (1) and (2) with (7) produces a solution that is characterized by Ekman balance by day and an inertial oscillation by night (initiated by the rapid decrease of the eddy viscosity at sunset); the oscillation can produce winds far in excess of the prevailing southerly geostrophic wind and thus explain the nocturnal low-level jet (Blackadar 1957). With $\alpha \neq 0$ and $K(t)$ a constant, the solution of (1)-(3) with (5) and (7) describes the response of a rotating stratified fluid with vertical diffusion and heat transfer to a diurnally varying surface temperature (Holton 1967). Both the Blackadar and Holton solutions are characterized by clockwise-turning hodographs, but differ in their predictions of the timing and amplitude of the low-level nocturnal wind maximum (see, e.g., $\mathrm{Du}$ and Rotunno 2014).

For the numerical solution of (1)-(6), the $z$ derivatives are discretized using centered differences with $\left(u_{k}, v_{k}, b_{k}\right)$ defined at grid points $z_{k}=(k-1 / 2) \Delta z$. Note that the drag-law and no-slip conditions are both applied at $z=0$. On the staggered vertical grid used in our numerical solutions, the prediction of velocity at the first grid level $(z=\Delta z / 2)$ involves the finite difference $\left[\tau_{x, y}(z=\Delta z)-\tau_{x, y}(z=0)\right] / d z$. In the

\footnotetext{
${ }^{1}$ Otherwise, the ratio of the periods of surface temperature increase to decrease would present yet another input parameter.
} 
no-slip case, $\tau_{x, y}(z=0)=K[(u, v)(z=\Delta z / 2)-(u, v)(z=$ $-\Delta z / 2)] / d z$, where the fictitious $(u, v)(z=-\Delta z / 2)=$ $-(u, v)(z=\Delta z / 2)$ to satisfy the no-slip condition at $z=0$; and in the drag-law case, $\tau_{x, y}(z=0)$ is given by (6). For the application of (6), we let $d=\Delta z / 2$; with $\Delta z=20 \mathrm{~m}$, the surface stress is evaluated using the $10-\mathrm{m}$ wind, as is standard. The top of the domain is put at $z=20 \mathrm{~km}$, so the infinite-depth analytical solutions of SFR may be well approximated. The time derivatives are also approximated by a centered difference, and the leapfrog method is used to advance the solution; a time step of $\Delta t=0.25 \mathrm{~s}$ was found to be adequate for all cases considered herein. As we are only interested in the timeperiodic solutions, we carry out the integrations until initial transients decay (approximately 4 days) and the diurnally periodic solution is found.

\section{b. Numerical solutions}

To test the present numerical solution, we use (7) and set the input parameters as specified in Table 1 of SFR $\left(f=8.6 \times 10^{-5} \mathrm{~s}^{-1}, v_{G}=10 \mathrm{~m} \mathrm{~s}^{-1}, \alpha=0.15^{\circ}\right.$, $N=0.01 \mathrm{~s}^{-1}, \Delta b=0.4 \mathrm{~m} \mathrm{~s}^{-2}, t_{\max }=9 \mathrm{~h}, t_{\mathrm{set}}=12 \mathrm{~h}, K_{d}=$ $100 \mathrm{~m}^{2} \mathrm{~s}^{-1}, K_{n}=1 \mathrm{~m}^{2} \mathrm{~s}^{-1}$, and $\delta=0.2$ day $^{-1}$ ). Comparing the hodograph in Fig. 1a with SFR's Fig. 6 (left panel) indicates that the present numerical solution gives a close match to SFR's analytical solution [their (3.17)]. ${ }^{2}$

Figure $1 \mathrm{~b}$ shows the hodographs from numerical solutions using the semislip condition (6) with $C_{D}=0.005$. For the levels displayed at $z \geq 110 \mathrm{~m}$, the hodographs turn clockwise with time, as in the no-slip case in Fig. 1a (but with reduced amplitude and a change in the time of maximum $v$ ). However, at the 10-m level, the hodograph behaviors of the no-slip and semislip cases are qualitatively different. In the no-slip case at $z=10 \mathrm{~m}$ (Fig. 1a), the orbit shrinks (eventually to a point as $z \rightarrow 0$ ) without changing turning direction, whereas in the semislip case, the hodograph tends to a finite orbit in the counterclockwise direction with sharp transitions after sunrise and sunset.

Figure 2 shows the variation with height of the hodograph in the semislip case as the counterclockwise turning changes to clockwise turning in the lowest $100 \mathrm{~m}$. The length scale of the variation with height is roughly the nighttime Ekman depth $\sqrt{K_{n} / 2 f}(\approx 77 \mathrm{~m})$, as seen in Fig. 2 and verified by further experimentation varying $K_{n}$ and $f$ (not shown).

The profiles in Fig. 3 illustrate the sunset transition $(12 \leq t \leq 21 \mathrm{~h})$ as shown in Fig. 5 of SFR: restricting

\footnotetext{
${ }^{2}$ Note that the noon icon in Fig. 1 denotes $t=6 \mathrm{~h}$, while in SFR's Fig. 6, the midday icon is placed at $t=t_{\max }=9 \mathrm{~h}$; we have also added an icon for midnight $(t=18 \mathrm{~h})$. Also the levels plotted are the model levels $z_{k}$ closest to the levels displayed in SFR's Fig. 6 a.
}
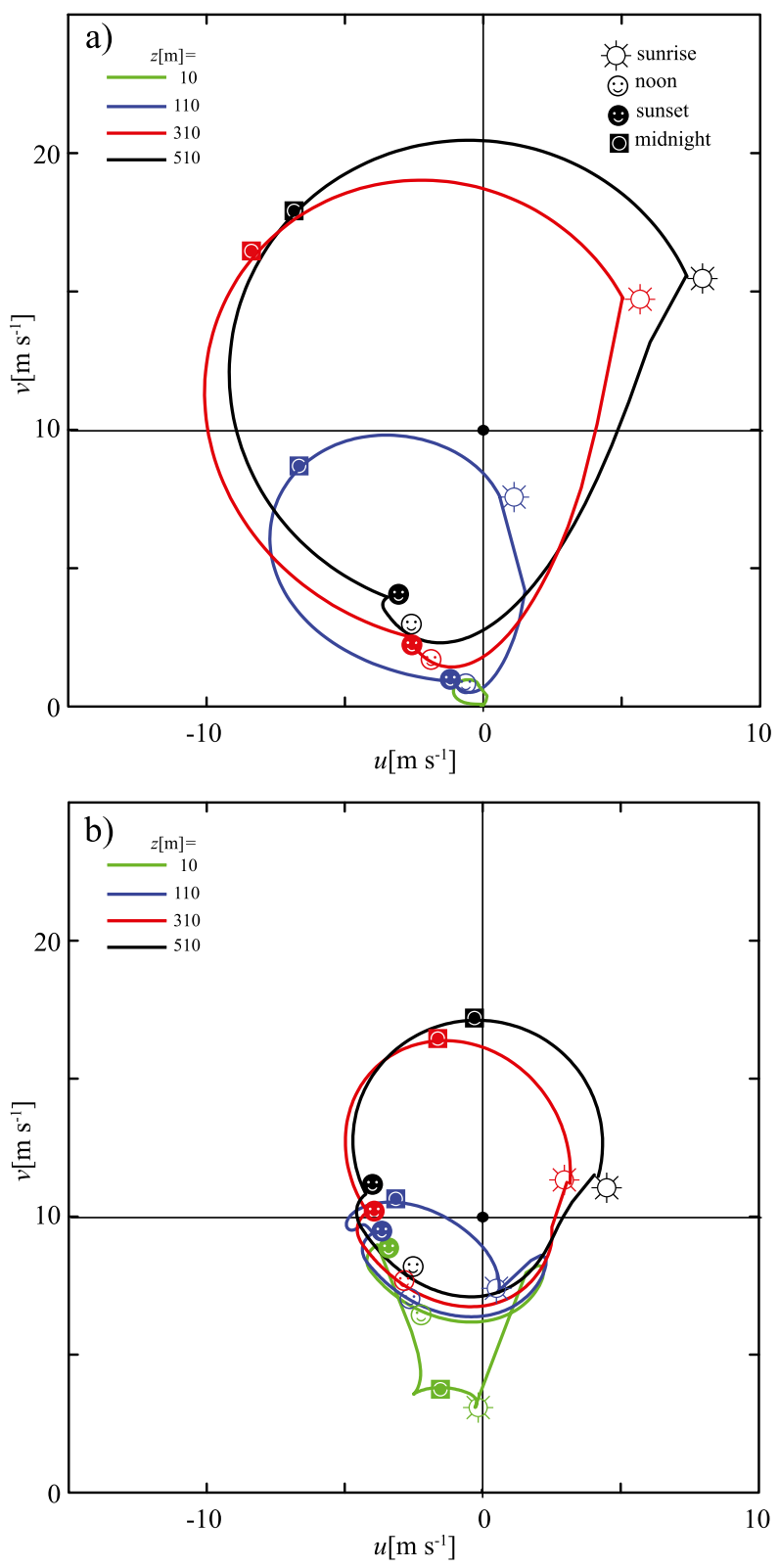

FIG. 1. Evolution of the wind hodographs at different heights with sunrise $(t=0 \mathrm{~h})$, noon $(t=6 \mathrm{~h})$, sunset $(t=12 \mathrm{~h})$, and midnight ( $t=18 \mathrm{~h}$ ) indicated by the icons for (a) the BH case of SFR's Fig. 6a and (b) the same case, but using a semislip lower-boundary condition. Note that the 10-m hodograph in (a) is not marked by icons. The geostrophic wind is marked by a dot at $(0,10) \mathrm{m} \mathrm{s}^{-1}$.

attention to $z<1 \mathrm{~km}$, the decrease in eddy viscosity after sunset in the no-slip case (Fig. 3a) initially leads to an increase of $-u$ and $v(t=15 \mathrm{~h})$ and then a decrease of $-u$ with $v$ increasing until roughly $t=20.5 \mathrm{~h}$ (not shown), consistent with an inertial oscillation. In this case, the time tendencies in $u$ and $v$ are qualitatively the same at each level. In the semislip case (Fig. 3b), the profile evolution after sunset in the layer extending from 


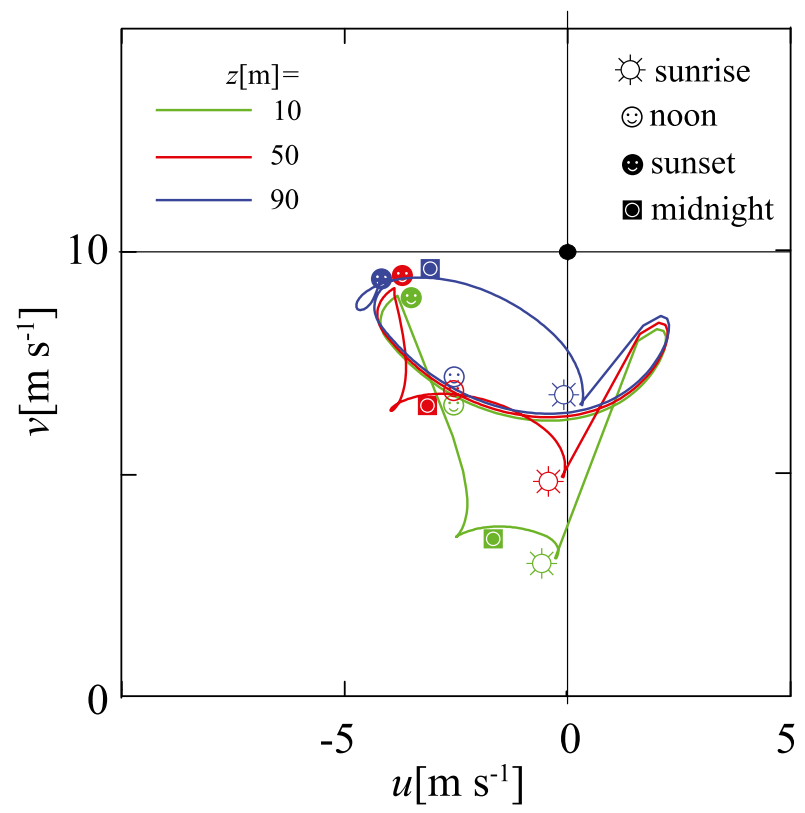

FIG. 2. As in Fig. 1, but at $z=10,50$, and $90 \mathrm{~m}$.

roughly $z \approx 100 \mathrm{~m} \mathrm{~m}$ to $z \approx 1 \mathrm{~km}$ is qualitatively the same as in the no-slip case (but again, we note the quantitative difference in the amplitude and time of the maximum $v$ ). Below $z \approx 100 \mathrm{~m}$, however, the effect of surface drag is seen to reduce $v$ immediately to roughly constant value, while $-u$ slowly decreases. Although there is surface drag in the no-slip case [ $=\left.K \partial_{z}(u, v)\right|_{z=0}$ ], it is proportional to $K(t)$, so its time changes are proportional to the interior stress; this is not the case with surface stress given by a drag law with an independent drag parameter as in (6). A more detailed examination of the terms in (1) and (2) follow.

Figure 4 shows the budget terms for the no-slip case at $z=10 \mathrm{~m}$ [all terms are nondimensionalized by $\omega v_{G}$ and the velocity $(u, v)$ by $\left.v_{G}\right]$. During the daytime $(t=0-12 \mathrm{~h})$, the balance of terms in Fig. 4 describes an Ekman-type balance modified by thermal forcing [at this low level, the velocity components are very small; thus, Fig. $4 \mathrm{~b}$ shows that all terms in (2) are small]. At sunset $(t=12 \mathrm{~h})$, the friction term in the $u$ equation abruptly decreases, and $u$ decelerates since $\partial u / \partial t \approx-f v_{G}-b \sin \alpha<0$; Fig. $4 \mathrm{~b}$ shows that in response to the postsunset $u<0$ (Fig. $4 a$ ), the terms $-f u$ and, therefore, $\partial v / \partial t$, increase, and thus an inertial oscillation is begun. At sunrise, friction reengages, and the daytime near-Ekman-type balance is restored. At the next higher level shown in Fig. $1 \mathrm{a}(z=110 \mathrm{~m})$, Fig. 5 indicates that the balances are qualitatively the same as at the lower level, but more smoothly varying in time.

In the semislip case at $z=10 \mathrm{~m}$, Fig. 6 shows that a thermally modified Ekman balance prevails during the daytime; however, at sunset, the abrupt decrease of $\left|\tau_{x}\right|$ above the surface indicates a decoupling of the interior and surface stresses, which induces a near discontinuity in the vertical stress profile $\left(\partial \tau_{x} / \partial z \approx-\left.\tau_{x}\right|_{z=10 \mathrm{~m}} / \Delta z \approx\right.$ $-\left.C_{D} v_{G} u\right|_{z=10 \mathrm{~m}} / \Delta z>0$, since $\left.u\right|_{z=10 \mathrm{~m}}<0$; see below), leading to a sharp acceleration of $u$ in contrast with the no-slip case (cf. Fig. 4a). Similarly, Fig. 6b shows a sharp deceleration of $v$ at sunset, in contrast with the no-slip case (cf. Fig. 4b). During the evening hours, Fig. 6a indicates the buoyancy term reverses sign and leads to a weak increase of $u$, while Fig. 6b shows that $v$ is nearly constant, consistent with Fig. 1b. At sunrise, the increase
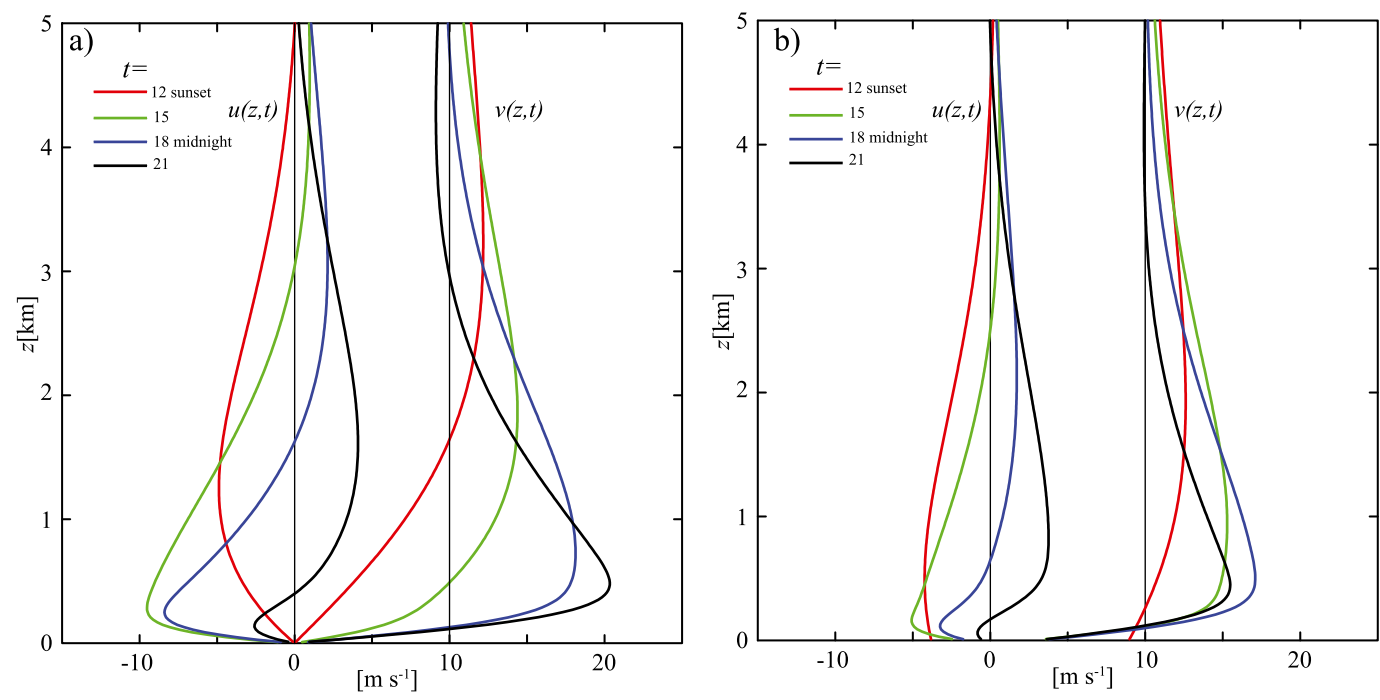

FIG. 3. Evolution of wind profiles after sunset for (a) the BH case of SFR's Fig. 5 and (b) the same case, but using a semislip lower-boundary condition. 

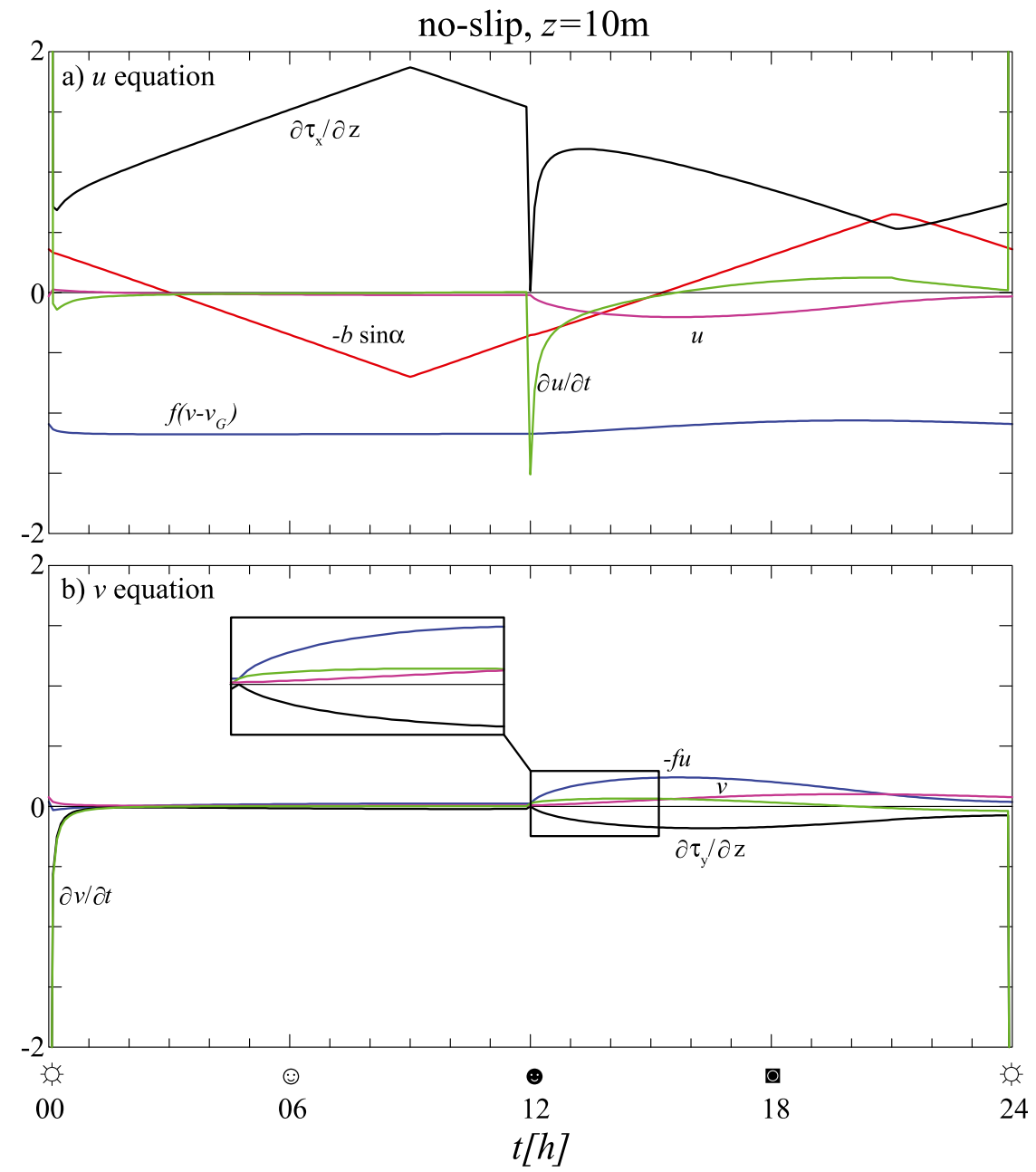

FIG. 4. Terms (nondimensionalized by $\omega v_{G}$ ) in (a) (1) and (b) (2) for the diurnally periodic solutions for the no-slip case at $z=10 \mathrm{~m}$. Velocity components $(u, v) / v_{G}$ are also plotted in their respective equations. A zoom box is added in (b) for extra clarity for the postsunset transition.

in both stress components in the interior leads to a recoupling of the interior and surface stresses and the restoration of the daytime near-Ekman balance.

In contrast with the no-slip case, the balance of terms in the semislip case is qualitatively different at higher levels. Figure 7 shows that at sunset, the acceleration due to the change in the stress profile is qualitatively consistent with the no-slip case at either level (Figs. 4, 5). And, as shown in Fig. 1b, the hodograph at $z=110 \mathrm{~m}$ turns in the clockwise direction.

The sunset transition for the semislip case is illustrated in detail in Fig. 8. Just before sunset $(t=11.9 \mathrm{~h})$, the stress-derivative components (red dashed lines) are nearly independent of $z$ over the lowest $100 \mathrm{~m}$. However, at sunset $(t=12.0 \mathrm{~h})$, the absolute values of the stress derivatives (green dashed lines) are large at the first grid point and zero above. Just after sunset $(t=12.1 \mathrm{~h})$, in response to the stress profiles at sunset, $-u$ and $v$ decrease, which, in turn, leads to a smoother variation of the stress profiles (blue dashed lines) with height.

Before leaving this section, we note that both the Blackadar and Holton mechanisms are needed to produce something resembling a counterclockwise-rotating hodograph at the surface. [The pure Blackadar (no slope with time-varying eddy viscosity) solution at the surface goes to a single point at night since there is no katabatic wind, while the pure Holton (slope with eddy viscosity constant in time) solution has clockwise turning at all levels since there is never a discontinuity in the vertical stress profile.] This result is consistent with Bluestein et al. (2018), in which both the observational analysis (their Fig. 5) and the time-averaged full-physics model simulations (their Fig. 12) indicate that counterclockwise turning with time of the surface hodographs only occurred 

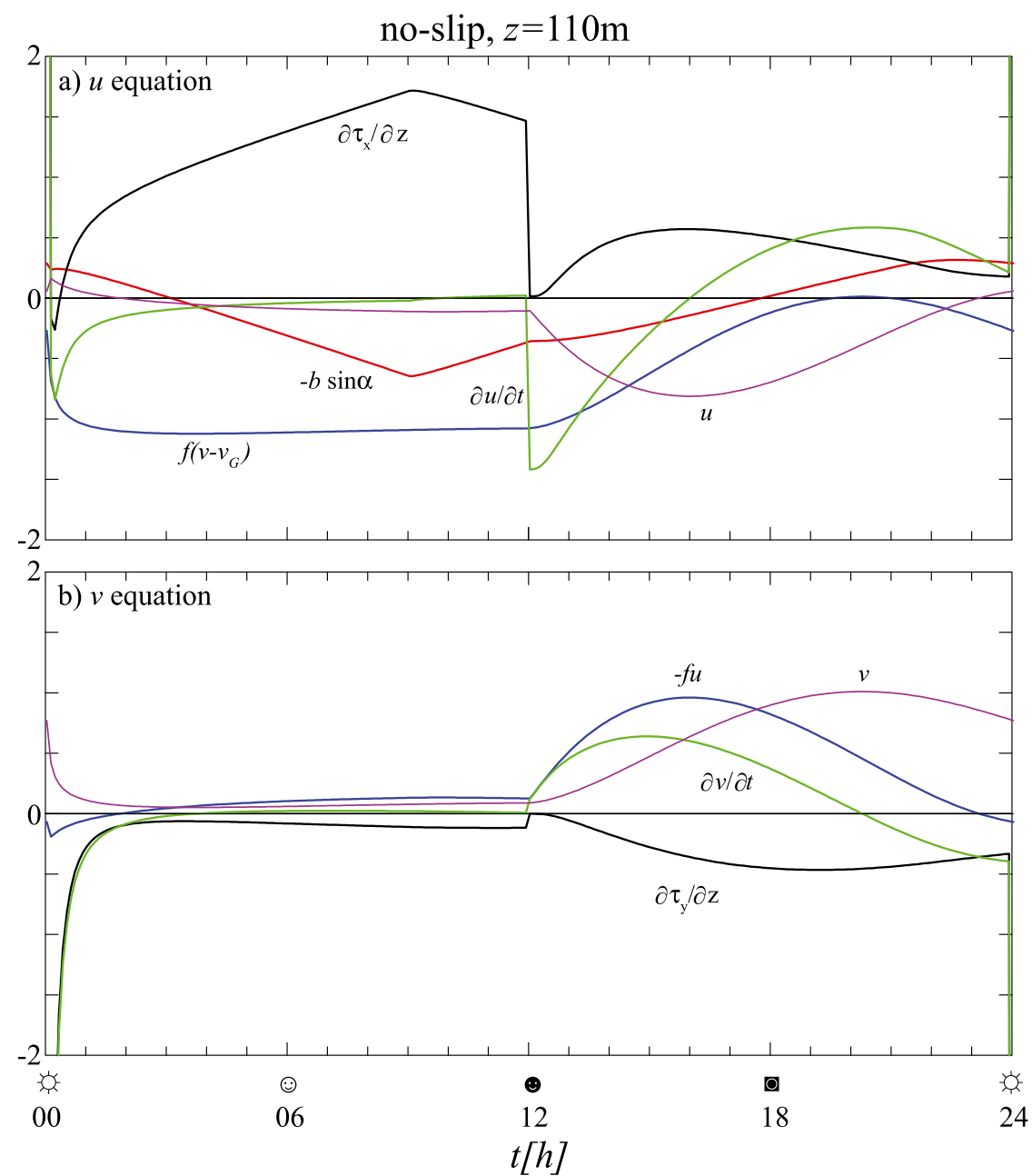

FIG. 5. As in Fig. 4, but at $z=110 \mathrm{~m}$.

on the gently sloping terrain roughly west of a line from Oklahoma City, Oklahoma, to Wichita, Kansas.

\section{Dimensional analysis and parameter dependence}

In their section $4 \mathrm{~d}$, SFR describe the main parameter dependence of their analytical solutions in terms of the maximum strength of the low-level jet, its height, and its time of occurrence. With the semislip condition (6), $C_{D}$ and, in principle, $d$ enter the list of external parameters that could influence the solution. Given the already-long list of external parameters, we are motivated to seek a reduced set of nondimensional parameters that most influence the solution.

\section{a. Dimensional analysis}

Within the confines of the present version of the SFR model (1)-(6), there are the following dimensional external parameters:

$$
v_{G}, \Delta b, K_{d}, K_{n}, f, \omega, N, t_{\max }, d, \delta
$$

in addition to the dimensionless parameters $\alpha$ and $C_{D}$. It was shown in SFR (and reproduced in the present study) that parameter $\delta$ has very little influence on the solutions, and thus, we neglect it from this point forward. Furthermore, by meteorological convention, $d=10 \mathrm{~m}$. Thus, not counting $d$ and $\delta,(8)$ indicates there are eight dimensional parameters. Since our equations involve two dimensions (length and time), dimensional analysis indicates that the eight dimensional parameters in (8) may be reduced to six dimensionless parameters; these six plus the dimensionless parameters $\alpha$ and $C_{D}$ govern the solutions. With some hindsight, we list the following dimensionless groups:

$$
\frac{K_{n}}{K_{d}}, \frac{\Delta b}{\omega v_{G}}, \frac{f}{\omega}, \frac{N}{\omega}, \frac{v_{G}}{\sqrt{\omega K_{d}}}, \omega t_{\max }
$$


semi-slip, $z=10 \mathrm{~m}$

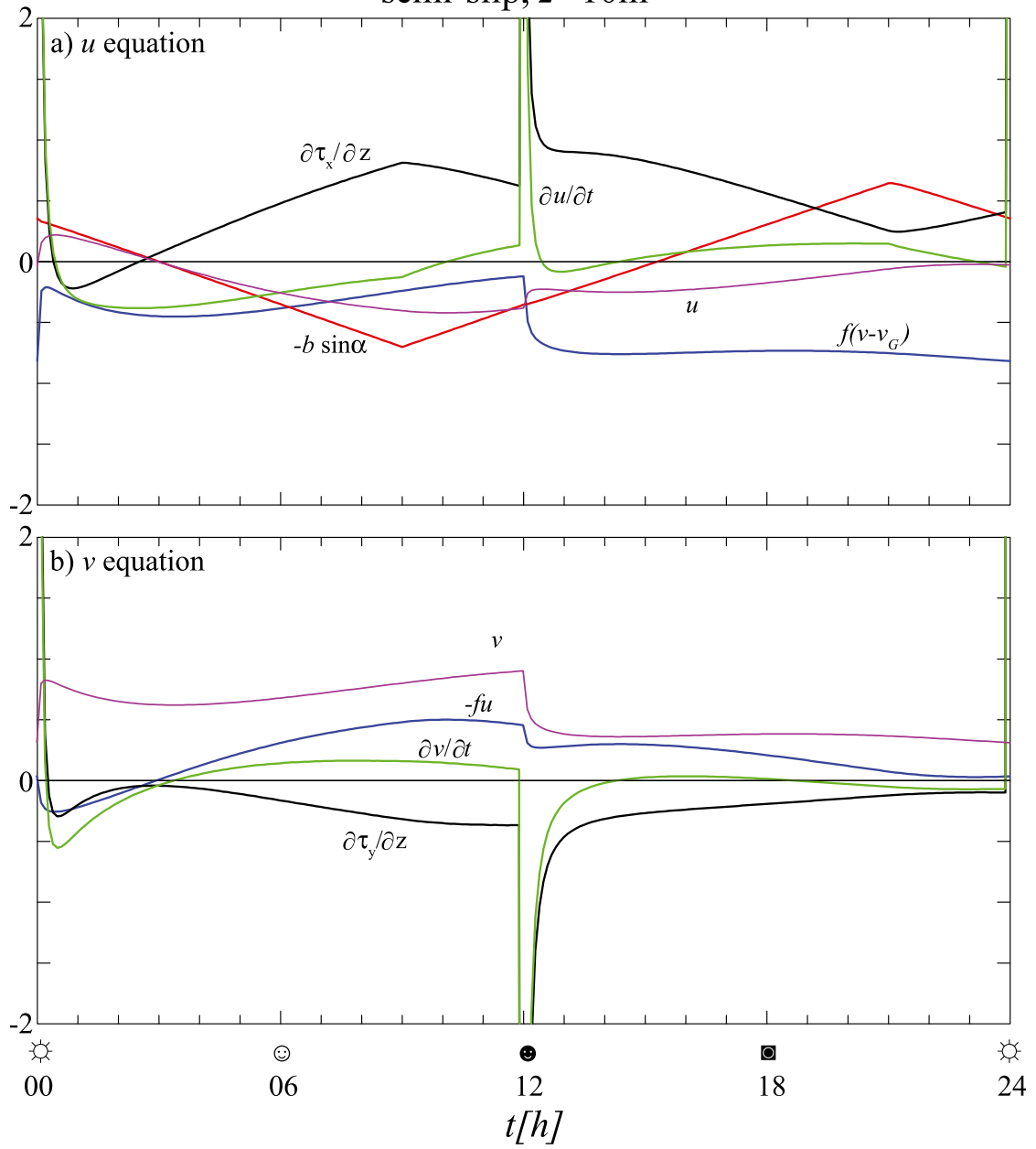

FIG. 6. As in Fig. 4, but for the semislip case.

in addition to $\alpha$ and $C_{D}$, as possible determinants of the solution.

\section{b. Nondimensional governing equations}

To obtain a dimensionless version of (1)-(6), we define

$$
\begin{aligned}
& \hat{t}=\omega t, \quad \hat{z}=z \sqrt{\frac{\omega}{K_{d}}}, \quad(\hat{u}, \hat{v})=\frac{(u, v)}{v_{G}}, \\
& \hat{b}=\frac{b}{\omega v_{G}}, \quad\left(\hat{\tau}_{x}, \hat{\tau}_{y}\right)=\frac{\left(\tau_{x}, \tau_{x}\right)}{v_{G}^{2}},
\end{aligned}
$$

which, upon substitution into (1)-(6), yields

$$
\begin{array}{ll}
\partial_{\hat{t}} \hat{u}=\frac{f}{\omega}(\hat{v}-1)-\hat{b} \sin \alpha & +\frac{v_{G}}{\sqrt{\omega K_{d}}} \partial_{\hat{z}} \hat{\tau}_{x}, \\
\partial_{\hat{t}} \hat{v}=-\frac{f}{\omega} \hat{u} & +\frac{v_{G}}{\sqrt{\omega K_{d}}} \partial_{\hat{z}} \hat{\tau}_{y},
\end{array}
$$

$$
\begin{aligned}
\partial_{\hat{t}} \hat{b} & =\hat{u} \frac{N^{2}}{\omega^{2}} \sin \alpha+\frac{K}{K_{d}} \partial_{\hat{z} \hat{z}} \hat{b}, \\
\left(\hat{\tau}_{x}, \hat{\tau}_{y}\right) & =\frac{K}{v_{G}} \sqrt{\frac{\omega}{K_{d}}} \partial_{\hat{z}}(\hat{u}, \hat{v}), \\
\left.\hat{b}\right|_{\hat{z}=0} & =\frac{1}{2} \frac{\Delta b}{\omega v_{G}} g(\hat{t}),
\end{aligned}
$$

and

$$
\left.\left(\hat{\tau}_{x}, \hat{\tau}_{y}\right)\right|_{\hat{z}=0}=\left.C_{D} \sqrt{\hat{u}^{2}+\hat{\boldsymbol{v}}^{2}}(\hat{u}, \hat{v})\right|_{\hat{z}=\hat{d}} .
$$

From (11)-(16), several properties of the solution parameter dependence can be inferred. First, substitution of (14) into (11) and (12) yields

$$
\frac{v_{G}}{\sqrt{\omega K_{d}}} \partial_{\hat{z}}\left(\hat{\tau}_{\hat{x}}, \hat{\tau}_{\hat{y}}\right)=\frac{K}{K_{d}} \partial_{\hat{z} \hat{z}}(\hat{u}, \hat{v})
$$


semi-slip, $z=110 \mathrm{~m}$

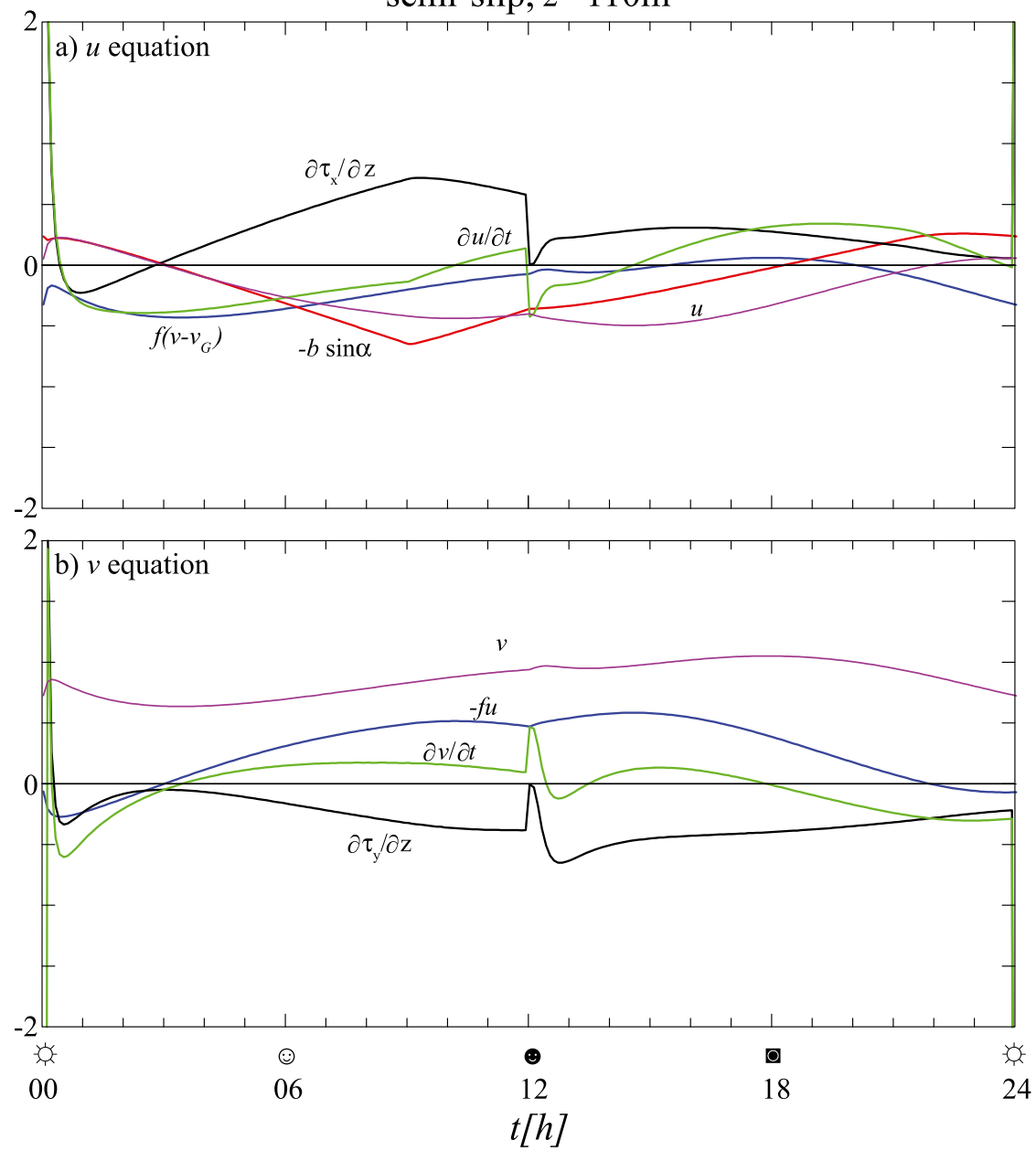

FIG. 7. As in Fig. 4, but for the semislip case at $z=110 \mathrm{~m}$.

for the friction terms. In the no-slip case, (17) applies at all levels, and therefore the only way that $v_{G}$ affects the solution $(\hat{u}, \hat{v}, \hat{b})$ is through the parameter $\Delta b / \omega v_{G}$; in the special (Blackadar) case where $\alpha=0$ (level ground) the solution for the velocity $(\hat{u}, \hat{v})$ is independent of $\Delta b / \omega v_{G}$. In the semislip case, if we consider the vertical integral of the friction terms in (11)-(12) from $\hat{z}=0$ to some height $\hat{z}>\hat{d}$, we find

$$
\begin{aligned}
\left.\frac{v_{G}}{\sqrt{\omega K_{d}}}\left(\hat{\tau}_{\hat{x}}, \hat{\tau}_{\hat{y}}\right)\right|_{\hat{z}=0} ^{\hat{\imath}>\hat{d}=} & \left.\frac{K}{K_{d}} \partial_{\hat{z}}(\hat{u}, \hat{v})\right|_{\hat{z}>\hat{d}} \\
& -\left.\frac{C_{D} v_{G}}{\sqrt{\omega K_{d}}} \sqrt{\hat{u}^{2}+\hat{v}^{2}}(\hat{u}, \hat{v})\right|_{\hat{z}=\hat{d}} .
\end{aligned}
$$

From (18), we see that the drag coefficient $C_{D}$ only appears in combination with $v_{G} / \sqrt{\omega K_{d}}$; therefore, $v_{G}$ influences the solution for $(\hat{u}, \hat{v})$ even when $\alpha=0$. In the limit as $C_{D} v_{G} / \sqrt{\omega K_{d}} \rightarrow \infty$, the velocity $\left.(\hat{u}, \hat{v})\right|_{\hat{z}=\hat{d}} \rightarrow 0$ to avoid infinite acceleration (owing to discontinuous stress); thus, the no-slip solutions are recovered in this limit.

The present analysis of the governing equations allows us to refine the space of nondimensional parameters (9) to

$$
\frac{K_{n}}{K_{d}}, \frac{\Delta b}{\omega v_{G}}, \frac{f}{\omega}, \frac{N}{\omega}, \frac{C_{D} v_{G}}{\sqrt{\omega K_{d}}}, \omega t_{\max }, \alpha .
$$

In the special case $\alpha=0$, the buoyancy drops out of (11), and the solution for $(\hat{u}, \hat{v})$ only depends on

$$
\frac{K_{n}}{K_{d}}, \frac{f}{\omega}, \frac{C_{D} v_{G}}{\sqrt{\omega K_{d}}} .
$$

We note that by definition, $f / \omega=2 \sin \phi$, where $\phi$ is the latitude. Since $\omega$ is a constant, the scale factor for time is the same for all experiments. In the computations to follow, we will keep $K_{d}=100 \mathrm{~m}^{2} \mathrm{~s}^{-1}$ so that the scale 


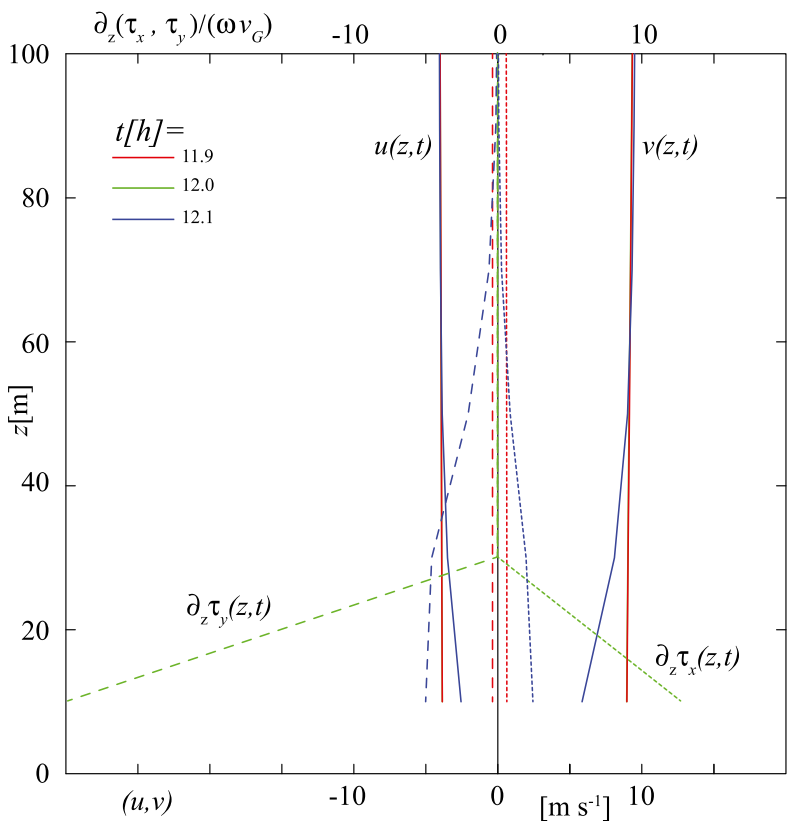

FIG. 8. Evolution of the wind and (nondimensional) stressderivative profiles close to the lower boundary at the sunset transition. (The velocity profiles at $t=12 \mathrm{~h}$ are hidden by those at $11.9 \mathrm{~h}$.) Long- and short-dashed lines correspond to the profiles $\partial_{z} \tau_{y}$ and $\partial_{z} \tau_{x}$, respectively. Note that the first grid point for the velocity and stress derivative is at $z=\Delta z / 2=10 \mathrm{~m}$.

factor for vertical distance $\sqrt{K_{d} / \omega} \approx 1172.8 \mathrm{~m}$ is the same for all experiments.

\section{c. Solution dependence on the nondimensional external parameters}

Each entry in Table 1 shows numerical solutions for $\hat{v}_{\max }$, its height $z_{\max }(\mathrm{m})$, and its time $t_{v_{\max }}(\mathrm{h})$ as a function of the three nondimensional parameters identified in (20) for the case with $\alpha=0$ (the Blackadar model). As mentioned above, the solution using the no-slip condition is recovered with $C_{D} v_{G} / \sqrt{\omega K_{d}} \rightarrow \infty$ and is given in the first two rows of Table 1 . The analytical solutions for the Blackadar cases reported in the second (case $\mathrm{B}$; Blackadar) and third (case $\mathrm{B}_{v_{G}^{+}}$; Blackadar with increased $v_{G}$ ) rows of Table 2 of SFR for $\alpha=0$ are for latitude $\phi=36.8^{\circ}$ and $K_{n} / K_{d}=0.01$, with $K_{d}=100 \mathrm{~m}^{2} \mathrm{~s}^{-1}$; for case $\mathrm{B}\left(v_{G}=10 \mathrm{~m} \mathrm{~s}^{-1}\right)$, the SFR results are $v_{\max }=16.8 \mathrm{~m} \mathrm{~s}^{-1}$ $\left(\hat{v}_{\max }=1.68\right), z_{\max }=460 \mathrm{~m}$, and $t_{v_{\max }}=21.0 \mathrm{~h}$, which are close $^{3}$ to those in Table 1 at $\phi=35^{\circ}$. The SFR results for case $B_{v_{G}^{+}} \quad\left(v_{G}=15 \mathrm{~m} \mathrm{~s}^{-1}\right), \quad v_{\max }=25.2 \mathrm{~m} \mathrm{~s}^{-1} \quad\left(\hat{v}_{\max }=1.68\right)$, $z_{\max }=460 \mathrm{~m}$, and $t_{v_{\max }}=21.0 \mathrm{~h}$, verify that the nondimensional velocity $(\hat{u}, \hat{v})$ is independent of $v_{G}$ in the

\footnotetext{
${ }^{3}$ With the SFR value of $\phi=36.8^{\circ}$, the present model reproduces values in case B of SFR's Table 1 to within its finite resolution.
}

no-slip case. It is generally recognized that $t_{v_{\max }}$ for the Blackadar model is somewhat later than found in the observations (Du and Rotunno 2014).

A few general features of the solution for $\alpha=0$ (Table 1) are as follows. The solution properties $\hat{v}_{\max }, z_{\max }$, and $t_{v_{\max }}$ all decrease with increasing $\phi$ for any value of $K_{n} / K_{d}$, as expected from past studies \{see Bonner and Paegle (1970, p. 743) for the $\phi$ dependence of the amplitude and phase and Shapiro and Fedorovich [2010, their (7)] for the $\phi$ dependence of the vertical scale\}. An increase in $K_{n} / K_{d}$ gives decreasing values of $\hat{v}_{\max }$ and $t_{v_{\max }}$ (more in accord with observations) but increasing $z_{\max }{ }^{4}$ The effect of the semislip condition (finite $C_{D} v_{G} / \sqrt{\omega K_{d}}$ ) is to decrease $\hat{v}_{\max }$ and $t_{v_{\max }}$ but only slightly affect $z_{\max }$. Note that as $C_{D} v_{G} / \sqrt{\omega K_{d}} \rightarrow 0$, $v(z, t)=v_{g}$ is a solution; hence, $\hat{v}_{\max } \rightarrow 1$, and $z_{\max }$ and $t_{v_{\max }}$ become indeterminate.

Turning to the case with $\alpha \neq 0$, the buoyancy term in (11) now plays a role in the solution for $(\hat{u}, \hat{v})$; therefore, all the nondimensional parameters in (19) may influence the solution. The analysis in Figs. 6 and 7 suggests that the buoyancy term in (11) is mostly determined by the surface forcing (5), and hence, we expect the parameter $\Delta b / \omega v_{G}$ to have a strong influence on the solution. In Table 2, the solution properties are listed for $\alpha=0.0025$ (characteristic of the Great Plains near western Oklahoma), $\Delta b / \omega v_{G}=550, N / \omega=0.01 \mathrm{~s}^{-1} /(2 \pi / 86400 \mathrm{~s})=136$, and $\omega t_{\max }=(2 \pi / 24 \mathrm{~h}) 9 \mathrm{~h}=3 \pi / 4$ and several values of $C_{D} v_{G} / \sqrt{\omega K_{d}}, K_{n} / K_{d}$, and $\phi$. As in the case with $\alpha=0$ (Table 1), Table 2 indicates that $\hat{v}_{\max }, z_{\max }$, and $t_{v_{\max }}$ all decrease with increasing latitude for any value of $K_{n} / K_{d}$ and $C_{D} v_{G} / \sqrt{\omega K_{d}}$, and an increase in $K_{n} / K_{d}$ gives decreasing values of $\hat{v}_{\max }$ and $t_{v_{\max }}$, but increasing $z_{\max }$. As $K_{n} / K_{d} \rightarrow 1$, the pure Holton case is approached. In this case, the $\hat{v}_{\max }$ are generally too small, and $\hat{t}_{v_{\max }}$ is too early, as compared to observations (Du and Rotunno 2014). Table 2 lists the results for $C_{D}=0$ and $K_{n} / K_{d} \rightarrow 1$; in this case, the solution represents a pure thermally forced oscillation in a rotating stratified fluid, as in the sea-breeze model of Rotunno (1983). As in the latter, the present model also exhibits a resonance at $\phi=30^{\circ}$ $(f / \omega=1)$.

Keeping $\alpha, N / \omega$, and $\omega t_{\max }$ the same as in Table 2, but letting $\Delta b / \omega v_{G}=733$, gives the results shown in Table 3 . For the no-slip cases $\left(C_{D} v_{G} / \sqrt{\omega K_{d}} \rightarrow \infty\right)$, the increase in $\Delta b / \omega v_{G}$ increases $\hat{v}_{\max }$ but leaves $z_{\max }$ and $t_{v_{\max }}$ largely unchanged. (We have verified that same solution occurs whether $\Delta b$ is increased or $v_{G}$ is decreased.) Increasing $\Delta b$ and $v_{G}$ so that $\Delta b / \omega v_{G}$ remains constant does not change the no-slip solutions. In Table 3 of SFR, the

\footnotetext{
${ }^{4}$ Note that these dimensional values will depend on $K_{d}$.
} 
TABLE 1. Each entry shows $\hat{v}_{\max }$, its height $z_{\max }(\mathrm{m})$, and its time $t_{v_{\max }}(\mathrm{h})($ midnight $=18 \mathrm{~h})$, separated by commas, as a function of $\phi\left({ }^{\circ}\right)$, $K_{n} / K_{d}$, and $C_{D} v_{G} / \sqrt{\omega K_{d}}$ for the case $\alpha=0$ (level terrain), which is the Blackadar case.

\begin{tabular}{|c|c|c|c|c|c|c|}
\hline \multirow[b]{2}{*}{$C_{D} v_{G} / \sqrt{\omega K_{d}}$} & \multirow[b]{2}{*}{$K_{n} / K_{d}$} & \multicolumn{5}{|c|}{$\phi$} \\
\hline & & $30^{\circ}$ & $35^{\circ}$ & $40^{\circ}$ & $45^{\circ}$ & $50^{\circ}$ \\
\hline$\infty$ & 0.01 & $1.74,510,22.5$ & $1.69,450,21.3$ & $1.69,430,20.4$ & $1.69,410,19.7$ & $1.69,390,19.1$ \\
\hline$\infty$ & 0.10 & $1.50,1490,20.4$ & $1.38,1250,19.4$ & $1.36,1150,18.9$ & $1.36,1070,18.5$ & $1.36,1030,18.0$ \\
\hline 1.17 & 0.01 & $1.44,550,20.3$ & $1.31,450,19.5$ & $1.30,410,19.0$ & $1.29,390,18.6$ & $1.28,370,18.1$ \\
\hline 1.17 & 0.10 & $1.30,1370,18.9$ & $1.19,1190,17.7$ & $1.17,1090,17.4$ & $1.16,990,17.4$ & $1.16,950,17.1$ \\
\hline 0.59 & 0.01 & $1.35,550,19.8$ & $1.23,450,19.0$ & $1.20,410,18.6$ & $1.20,370,18.5$ & $1.19,350,18.1$ \\
\hline 0.59 & 0.10 & $1.23,1330,18.6$ & $1.13,1170,17.3$ & $1.12,1070,17.1$ & $1.12,970,17.3$ & $1.11,930,17.0$ \\
\hline
\end{tabular}

Blackadar-Holton experiment $(\mathrm{BH})$ has $v_{\max }=21.1 \mathrm{~m} \mathrm{~s}^{-1}$, $z_{\max }=460 \mathrm{~m}$, and $t_{v_{\max }}=21.0 \mathrm{~h}$ for input values of $\Delta b=0.2 \mathrm{~m} \mathrm{~s}^{-2}$ and $v_{G}=10 \mathrm{~m} \mathrm{~s}^{-2}$; we infer that $\hat{v}_{\max }=2.11$. SFR (p. 3051) report a case in which $\Delta b=0.3 \mathrm{~m} \mathrm{~s}^{-2}$ and $v_{G}=15 \mathrm{~m} \mathrm{~s}^{-2}$ and find $v_{\max }=32 \mathrm{~m} \mathrm{~s}^{-1}$. Since $\Delta b / \omega v_{G}$ is unchanged from their $\mathrm{BH}$ case, we expect that $v_{\max }=2.11 \times 15 \mathrm{~m} \mathrm{~s}^{-1} \approx 32 \mathrm{~m} \mathrm{~s}^{-1}$, which is consistent with SFR's result. The semislip results shown in Table 3 show the same general tendencies with respect to the no-slip cases, as seen in Table $1(\alpha=0)$ or Table 2 $\left(\Delta b / \omega v_{G}=550\right)$.

The present calculations reproduce the dependence of $\hat{v}_{\max }$ on $\alpha$, as described in Fig. 9 of SFR (peaking at $\alpha \approx 0.25^{\circ}$ ) for either no-slip or semislip conditions (although the peak occurs for larger $\alpha$ ). Finally, increasing $t_{\max }$ to sunset increases $\hat{v}_{\max }$ for either no-slip (as found in SFR) or semislip conditions.

\section{Conclusions}

Surface hodographs over the Great Plains exhibit anomalous counterclockwise turning over the diurnal cycle, which is opposite of the expected clockwise turning based on higher-level observations and theory; mesoscale-model forecasts over the continental United States from the warm season exhibit the same behavior (Bluestein et al. 2018). Analysis of the mesoscale-model forecasts in Bluestein et al. (2018) revealed that at sunset and sunrise, sharp vertical gradients in the stress profiles occur due to the continuous action of the surface stress and the decay and growth of interior stress due to the diurnal cycle of turbulence. The present paper extends the simple onedimensional model of Shapiro et al. (2016) to include a semislip (instead of a no-slip) lower-boundary condition and is able to qualitatively reproduce the anomalous counterclockwise turning over the diurnal cycle in the surface layer while retaining the expected clockwise turning at higher levels.

The semislip lower boundary condition, in addition to its effect on the direction of turning in the surface layer, also produces quantitative changes in the solutions at all levels. As the number of potentially important input parameters in the extended SFR model is large, we reduced that number by looking for the most important dependence of the solution on several dimensionless input parameters. In summary, the nondimensional parameters that influence the no-slip or semislip solutions to the SFR model are given by (19). A novel result is the identification of the control parameter $\Delta b / \omega v_{G}$ for which increasing values give increasing $v_{\max } / v_{G}$; in the no-slip limit $\left(C_{D} v_{G} / \sqrt{\omega K_{d}} \rightarrow \infty\right), \Delta b / \omega v_{G}$ is the only one involving $v_{G}$. The effect of the semislip parameter $C_{D} v_{G} / \sqrt{\omega K_{d}}$ is to reduce $v_{\max } / v_{G}$ and $t_{v_{\max }}$ with only a slight effect on $z_{\max }$. A practical consequence of the present analysis is that one would expect that during the height of the warm season, when the difference between the daytime and nighttime buoyancy is greatest, the meridional component

TABLE 2. As in Table 1, but for the case $\alpha=0.0025$ (sloping terrain) and $\Delta b / \omega v_{G}=550, N / \omega=136$, and $\omega t_{\max }=3 \pi / 4\left(t_{\max }=9 \mathrm{~h}\right)$.

\begin{tabular}{|c|c|c|c|c|c|c|}
\hline \multirow[b]{2}{*}{$C_{D} v_{G} / \sqrt{\omega K_{d}}$} & \multirow[b]{2}{*}{$K_{n} / K_{d}$} & \multicolumn{5}{|c|}{$\phi$} \\
\hline & & $30^{\circ}$ & $35^{\circ}$ & $40^{\circ}$ & $45^{\circ}$ & $50^{\circ}$ \\
\hline$\infty$ & 0.01 & $2.13,550,21.7$ & $2.07,490,20.8$ & $2.04,470,19.9$ & $2.02,430,19.3$ & $2.01,410,18.8$ \\
\hline$\infty$ & 0.10 & $1.79,1410,19.7$ & $1.68,1230,19.1$ & $1.64,1150,18.5$ & $1.63,1090,18.0$ & $1.61,1050,17.7$ \\
\hline$\infty$ & 0.99 & $1.19,3510,18.5$ & $1.15,3150,16.9$ & $1.14,2970,16.1$ & $1.14,2830,15.6$ & $1.13,2730,15.3$ \\
\hline 0 & 0.99 & No steady solution & $1.54,-, 12.7$ & $1.5,2-, 12.9$ & $1.34,-, 12.4$ & $1.37,-, 12.1$ \\
\hline 1.17 & 0.01 & $1.98,590,19.6$ & $1.79,510,19.0$ & $1.72,470,18.3$ & $1.68,450,17.9$ & $1.64,410,17.5$ \\
\hline 1.17 & 0.10 & $1.77,1290,18.3$ & $1.58,1130,17.6$ & $1.52,1070,17.0$ & $1.49,1030,16.7$ & $1.46,970,16.4$ \\
\hline 1.17 & 0.99 & $1.35,2630,17.2$ & $1.25,2210,15.4$ & $1.23,2070,14.6$ & $1.20,1970,14.2$ & $1.19,1890,13.8$ \\
\hline
\end{tabular}


TABLE 3. As in Table 1, but for the case $\alpha=0.0025$ (sloping terrain) and $\Delta b / \omega v_{G}=733, N / \omega=136$, and $\omega t_{\max }=3 \pi / 4\left(t_{\max }=9 \mathrm{~h}\right)$.

\begin{tabular}{|c|c|c|c|c|c|c|}
\hline \multirow[b]{2}{*}{$C_{D} v_{G} / \sqrt{\omega K_{d}}$} & \multirow[b]{2}{*}{$K_{n} / K_{d}$} & \multicolumn{5}{|c|}{$\phi$} \\
\hline & & $30^{\circ}$ & $35^{\circ}$ & $40^{\circ}$ & $45^{\circ}$ & $50^{\circ}$ \\
\hline$\infty$ & 0.01 & $2.33,570,21.7$ & $2.24,510,20.7$ & $2.19,470,19.9$ & $2.16,450,19.3$ & $2.14,430,18.8$ \\
\hline$\infty$ & 0.10 & $1.95,1410,19.7$ & $1.81,1250,19.0$ & $1.76,1170,18.4$ & $1.73,1110,18.0$ & $1.72,1070,17.6$ \\
\hline 1.17 & 0.01 & $2.20,610,19.7$ & $1.97,510,19.0$ & $1.87,470,18.3$ & $1.82,450,17.9$ & $1.77,430,17.5$ \\
\hline 1.17 & 0.10 & $1.95,1290,18.4$ & $1.73,1130,17.6$ & $1.65,1070,17.0$ & $1.61,1030,16.7$ & $1.57,970,16.4$ \\
\hline
\end{tabular}

of the wind at a given latitude is most supergeostrophic. Factors that could also affect the degree to which $v$ is supergeostrophic include the nature of the underlying vegetation and the wetness of the soil, which could affect the buoyancy. Since low-level vertical shear may be increased as $v$ increases in speed, one would expect the intensity of convective storms that might form would also increase as the soil wetness decreases or if vegetation becomes sparser.

Acknowledgments. H. Bluestein is supported by NSF Grant AGS-1560945. We thank Prof. Yu Du (Sun Yat-sen University, China) for his comments on the first draft of this paper.

\section{REFERENCES}

Blackadar, A. K., 1957: Boundary layer wind maxima and their significance for the growth of nocturnal inversions. Bull. Amer. Meteor. Soc., 38, 283-290.

Bluestein, H. B., G. S. Romine, R. Rotunno, D. W. Reif, and C. C. Weiss, 2018: On the anomalous counterclockwise turning of the surface wind with time in the plains of the United States.
Mon. Wea. Rev., 146, 467-484, https://doi.org/10.1175/MWRD-17-0297.1.

Bonner, W. D., and J. Paegle, 1970: Diurnal variations in boundary layer winds over the south-central United States in summer. Mon. Wea. Rev., 98, 735-744, https://doi.org/10.1175/15200493(1970)098<0735:DVIBLW >2.3.CO;2.

Du, Y., and R. Rotunno, 2014: A simple analytical model of the nocturnal low-level jet over the Great Plains of the United States. J. Atmos. Sci., 71, 3674-3683, https://doi.org/10.1175/JAS-D-140060.1 .

Glickman, T., Ed., 2000: Glossary of Meteorology. 2nd ed. Amer. Meteor. Soc., 855 pp., http://glossary.ametsoc.org/.

Holton, J. R., 1967: The diurnal boundary layer wind oscillation over sloping terrain. Tellus, 19A, 199-205, https://doi.org/ 10.1111/j.2153-3490.1967.tb01473.x.

Lumley, J. L., and H. A. Panofsky, 1964: The Structure of Atmospheric Turbulence. Wiley, $239 \mathrm{pp}$.

Rotunno, R., 1983: On the linear theory of the land and sea breeze. J. Atmos. Sci., 40, 1999-2009, https://doi.org/10.1175/15200469(1983)040<1999:OTLTOT>2.0.CO;2.

Shapiro, A., and E. Fedorovich, 2010: Analytical description of a nocturnal low-level jet. Quart. J. Roy. Meteor. Soc., 136, 12551262, https://doi.org/10.1002/qj.628.

- , and S. Rahimi, 2016: A unified theory for the Great Plains nocturnal low-level jet. J. Atmos. Sci., 73, 3037-3057, https://doi.org/10.1175/JAS-D-15-0307.1. 